\title{
The Total Ground State Energies and First Ionization Energies of the Incomplete 3d-Transition Metal-Elements Atoms
}

\author{
Ebtehal M. Althobaiti ${ }^{1}$, Atif Ismail ${ }^{1,2, *}$, M. Sabry ${ }^{1,3}$ \\ ${ }^{1}$ Physics Department, College of Applied Science, Umm Al Qura University, Makkah, Saudi Arabia \\ ${ }^{2}$ Physics Department, Faculty of Science, Kafrelsheikh University, Kafr El-Sheikh, Egypt \\ ${ }^{3}$ National Research Institute of Astronomy and Geophysics, Cairo, Egypt
}

Copyright $@ 2017$ by authors, all rights reserved. Authors agree that this article remains permanently open access under the terms of the Creative Commons Attribution License 4.0 International License

\begin{abstract}
We studied the incomplete 3d-transition metal-lements, scandium through nikel, atoms and their corresponding cations by diffusion Monte Carlo (DMC) method with three different basis sets, namely VTZ_ANO, Stuttgart RSC 1997 ECP, and CRENBL ECP. Our calculations for total ground state energy and first ionization potential, agree very well with studies used LANL2DZ $\mathrm{B}_{\mathrm{B} L Y P}$ as basis sets, and with the experimental values of first ionization potential. Moreover, we found that the calculations with VTZ_ANO basis set are more accurate than those with the other two basis sets.
\end{abstract}

Keywords Diffusion Monte Carlo Method, Total Ground State Energy, First Ionization Potential

\section{Introduction}

The transition metals play important roles in modern technology [1]. There are three noteworthy elements in the transition metals family: iron, cobalt, and nickel. They are the only elements known to produce a magnetic field [2], for their interresting properties so, there is a reason to study them, beside the sake of comparison with others results.

Quantum Monte Carlo methods (e.g. VMC and DMC methods) are commonly applied in studying and calculating the properties and physical quantities of single atoms, molecules, and compounds and solids.

Also, there are some efficient software codes used for quantum chemistry and quantum Monte Carlo calculations like gamess [3] and Qwalk [4].

Our study is aiming to the following: First the examination of how reliable, accurate, and adoptable and adaptable DMC method. Second make a comparison between the three basis sets. Third, to examine how accurate our results for ground state energy and ionization potential by comparing them with other theoretical and experimental results from literature.

\section{Computational Method}

Monte Carlo means using random numbers in scientific computing. For example, let $X$ be a random variable and write its expected value as $A=E[X]$. If we can generate $X_{1}, \ldots, X_{N}, N$ independent random variables with the same distribution, then we can make the approximation:

$$
A \approx \hat{A}_{N}=\frac{1}{N} \sum_{i=1}^{N} X_{i},
$$

where $X$ is a random variable and $A=E[X]$ its expected value.

The strong law of large numbers states that $\hat{A}_{N} \rightarrow A$ as $N \rightarrow \infty$. The $X_{i}$ and $\hat{A}_{N}$ are random but $A$ is not [5].

The diffusion Monte Carlo (DMC) method is one of the real-space stochastic projector techniques and is a key tool in electronic-structure Quantum Monte Carlo (QMC). It is based on solving the imaginary time many-body Schrödinger equation:

$$
\begin{aligned}
-\frac{\partial \Psi(\mathbf{R}, \tau)}{\partial \tau} & =H \Psi(\mathbf{R}, \tau) \\
& =D \nabla^{2} \Psi(\mathbf{R}, \tau)+\left(E_{T}-\hat{V}\right) \Psi(\mathbf{R}, \tau),
\end{aligned}
$$

where $\tau$ is the imaginary time, $\Psi$ is a solution and $E_{T}$ is an energy offset. The RHS of (2) is the sum of diffusion and rate terms. One may formally expand the solution in a complete set of the eigenfunctions $\left(\Psi_{n}\right)$ with the corresponding energies $\left(E_{n}\right)$,

$$
\begin{aligned}
\Psi(\mathbf{R}, \tau) & =\sum_{n=0}^{\infty} C_{n} \Psi_{n}(\mathbf{R}) e^{-\left(E_{n}-E_{0}\right) \tau} \\
& =C_{0} \Psi_{0}+\sum_{n=1}^{\infty} C_{n} \Psi_{n}(\mathbf{R}) e^{-\left(E_{n}-E_{0}\right) \tau} .
\end{aligned}
$$

Now all states with energy above $E_{T}$ decay, and those with energy below $E_{T}$ are amplified in (imaginary) time [6]. 
The operator responsible for the time evolution is the Green's function $\hat{G}=e^{-\tau\left(H-E_{T}\right)}$ which satisfies the operator equation [7]:

$$
-\frac{\partial \hat{G}}{\partial \tau}=\left(\hat{H}-E_{T}\right) \hat{G}
$$

Green's function which contains the projection operator and satisfies (2) is given by:

$$
\begin{aligned}
G\left(\mathbf{R} \leftarrow \mathbf{R}^{\prime}, \delta \tau\right)= & \left\langle\mathbf{R}\left|e^{-\delta \tau\left(H-E_{T}\right)}\right| \mathbf{R}^{\prime}\right\rangle \approx e^{-\delta \tau\left[\hat{V}(\mathbf{R})-E_{T}\right] / 2} \\
& \left\langle\mathbf{R}\left|e^{-\delta \tau \hat{T}}\right| \mathbf{R}^{\prime}\right\rangle e^{-\delta \tau\left[\hat{V}\left(\mathbf{R}^{\prime}\right)-E_{T}\right] / 2}
\end{aligned}
$$

A possible and useful form of the approximate Green's function correct for $\delta \tau \rightarrow 0$ contains the known solutions to the diffusion and rate equation, respectively:

$$
\begin{aligned}
G\left(\mathbf{R} \leftarrow \mathbf{R}^{\prime}, \delta \tau\right) \approx & (2 \pi \delta \tau)^{-3 N / 2} e^{-\frac{\left(\mathbf{R}-\mathbf{R}^{\prime}\right)^{2}}{2 \delta \tau}} \\
& \times e^{-\delta \tau\left[\hat{V}(\mathbf{R})+\hat{V}\left(\mathbf{R}^{\prime}\right)-2 E_{T}\right] / 2},
\end{aligned}
$$

and the error is proportional to $\delta \tau^{3}$. The second exponential factor acts as a renormalization and accomplishes the birth/death process. The population of walkers fluctuates around the desired mean as it is controlled by $E_{T}[6,8]$.

In a DMC simulation, one treats $\Psi(\mathbf{R}, \tau)$ as the density distribution of a large ensemble of equivalent copies of the many body system, usually called walkers. The simulation starts with an arbitrary initial distribution and the population of walkers diffuses according to the Green function. The first term corresponds to free particle diffusion, but the second term instead does not cause displacement of particles. It determines only a change in the probability density. This effect, usually called "branching", can be implemented by allowing variations in the number of walkers [9].

The renormalization

factor $\exp \left[-\delta \tau\left[\hat{V}(\mathbf{R})+\hat{V}\left(\mathbf{R}^{\prime}\right)-2 E_{T}\right] / 2\right]$ in (6) causes large fluctuations of the population and so does the local energy, from step to step, since the rate term diverges whenever two electrons or electron and nucleus approach too close to each other. This leads to an extremely poor statistical behavior and makes the renormalization process "ill defined". These difficulties may be overcome by the importance-sampling transformation provided with help of a guiding wave function $\Psi_{T}(\mathbf{R})$ that must be supplied as an input to the DMC method. If we consider the mixed distribution $f(\mathbf{R}, \tau)=\Psi(\mathbf{R}, \tau) \Psi_{T}(\mathbf{R})$ and substitute it into (2) multiplied by $\Psi_{T}(\mathbf{R})$, we obtain equation in the form

$$
\begin{aligned}
-\frac{f(\mathbf{R}, \tau)}{\partial \tau}= & -\frac{1}{2} \nabla^{2} f(\mathbf{R}, \tau)+\nabla \cdot\left[\mathbf{v}_{D}(\mathbf{R}) f(\mathbf{R}, \tau)\right] \\
& +\left[E_{L}(\mathbf{R})-E_{T}\right] f(\mathbf{R}, \tau),
\end{aligned}
$$

where $\mathbf{v}_{D}(\mathbf{R})$ is the $3 \mathrm{~N}$-dimensional drift velocity defined by:

$$
\mathbf{v}_{D}(\mathbf{R})=\nabla \ln \left|\Psi_{T}(\mathbf{R})\right|=\frac{\nabla \Psi_{T}(\mathbf{R})}{\Psi_{T}(\mathbf{R})}
$$

and

$$
E_{L}(\mathbf{R})=\Psi_{T}(\mathbf{R})^{-1} \hat{H} \Psi_{T}(\mathbf{R})
$$

is the local energy. This formulation implicitly imposes the Fixed Node (FN) approximation.

The FN-DMC energy estimator is given by:

$$
E_{\mathrm{DMC}}=\frac{\int f(\mathbf{R}) E_{L}(\mathbf{R}) \mathrm{d} \mathbf{R}}{\int f(\mathbf{R}) \mathrm{d} \mathbf{R}}
$$

which tends to the exact value with an increasing accuracy of the nodal surface of $\Psi_{T}$. The FN-DMC algorithm is highly accurate and satisfies the zero-variance zero-bias principle, i.e. the variance of the energy and its bias goes to zero as the trial wave function approaches the exact eigenstate $[6,8]$.

Expectation value of the energy can be evaluated as the mixed estimator. This is given (which is similar to (1) as expected.) by

$$
\begin{aligned}
E_{\mathrm{DMC}} & =\lim _{\tau \rightarrow \infty} \frac{\left\langle e^{-\tau \hat{H} / 2} \Psi_{T}|\hat{H}| e^{-\tau \hat{H} / 2} \Psi_{T}\right\rangle}{\left\langle e^{-\tau \hat{H} / 2} \Psi_{T} \mid e^{-\tau \hat{H} / 2} \Psi_{T}\right\rangle} \\
& =\lim _{\tau \rightarrow \infty} \frac{\left\langle e^{-\tau \hat{H}} \Psi_{T}|\hat{H}| \Psi_{T}\right\rangle}{\left\langle e^{-\tau \hat{H}} \Psi_{T} \mid \Psi_{T}\right\rangle}=\frac{\left\langle\Psi_{0}|\hat{H}| \Psi_{T}\right\rangle}{\left\langle\Psi_{0} \mid \Psi_{T}\right\rangle} \\
& =\lim _{\tau \rightarrow \infty} \frac{\int f(\mathbf{R}, \tau) E_{L}(\mathbf{R}) \mathrm{d} \mathbf{R}}{\int f(\mathbf{R}, \tau) \mathrm{d} \mathbf{R}} \\
& \approx \frac{1}{N} \sum_{i} E_{L}\left(\mathbf{R}_{i}\right)
\end{aligned}
$$

where $\mathbf{R}_{i}$ is the set of $N$ samples from the DMC run [8].

In what follows, we present the numerical results for incomplete 3d-transtion elements, scandium through nikel, atoms and their corresponding cations. These numerical results were obtained by means of Qwalk code [4]. The basic form of the wavefunction consists of a product of Slater determinants for spin-up and spin-down electrons multiplied by a Jastrow correlation factor. The initial orbital of the trial wavefunction are generated by means of GAMESS package [3] via spin-restricted open Hartree-Fock calculations [9]. Of cource, the interesting physics is usually in the valence region and on the energy scale of the valence electrons [12]. Thus, pseudopotential calculations were performed for all the studied atoms since QMC calculations involving all the atomic electrons are not practical for these heavy atoms [9].

\section{Results And Discussion}

\subsection{The total energy}

The energy is obtained very accurately in DMC because the local energy is nearly constant with proper importance function. However, other properties, not commuting with the Hamiltonian have a possible systematic bias because averages are obtained from the mixed distribution $f=\phi \Psi$ where $\phi$ is the exact wavefunction instead of from the square of the ground state wavefunction [12]. 
Table 1. The total ground state energies computed within Hartree Fock, $E_{H F}$, variational Monte Carlo, $E_{V M C}$, and diffusion Monte Carlo, $E_{\mathrm{DMC}}$, for $\mathrm{Sc}$ through Ni compared to $E_{D M C}\left(L A N L 2 D Z_{\mathrm{B} 3 \mathrm{LYP}}\right)$ results calculated by Chiodo et al. [13]. The statistical error is given in parenthesis. All energies are in Hartree $(\mathrm{H})$.

\begin{tabular}{|c|c|c|c|c|c|}
\hline Basis Set & Atom & $\mathrm{E}_{\mathrm{HF}}$ & $\mathrm{E}_{\mathrm{VMC}}$ & $\mathrm{E}_{\mathrm{DMC}}(\sigma)$ & $\begin{array}{c}\mathrm{E}_{\mathrm{DMC}} \\
\left(\mathrm{LANL2DZ}{ }_{\mathrm{B} 3 \mathrm{LYP}}\right)\end{array}$ \\
\hline STUTTGART & & -46.20710483 & -46.51672427 & $-46.62998863(0.98)$ & \\
\hline CRENBL ECP & & -45.97198402 & -46.2909601 & $-46.48018627(1.69)$ & \\
\hline VTZ_ANO & Sc & -46.1917592 & -46.48995954 & $-46.7093261(0.78)$ & -46.3778756 \\
\hline STUTTGART & & -57.80807275 & -58.0596808 & $-58.16362738(1.61)$ & \\
\hline CRENBL ECP & & -57.53150634 & -57.8300806 & $-57.93683152(1.31)$ & \\
\hline VTZ_ANO & $\mathrm{Ti}$ & -57.32830944 & -58.12790402 & $-58.18668811(0.87)$ & -57.9887518 \\
\hline STUTTGART & & -70.90607295 & -71.43743143 & $-71.51737501(5.08)$ & \\
\hline CRENBL ECP & & -70.58193748 & -71.23774982 & $-71.2433229(1.61)$ & \\
\hline VTZ_ANO & $\mathrm{V}$ & -71.18341399 & -71.48679066 & $-71.5606345(1.00)$ & -71.2393538 \\
\hline STUTTGART & & -86.2829349 & -86.5640338 & $-86.87485318(1.95)$ & \\
\hline CRENBL ECP & & -85.91343006 & -86.40487202 & $-86.52876152(1.93)$ & \\
\hline VTZ_ANO & $\mathrm{Cr}$ & -86.52474198 & -86.81076609 & $-87.00410452(1.10)$ & -86.2727611 \\
\hline STUTTGART & & -102.9870621 & -103.9470537 & $-105.8612472(2.33)$ & \\
\hline CRENBL ECP & & -103.2104178 & -103.4982319 & $-104.5486169(2.20)$ & \\
\hline VTZ_ANO & $\mathrm{Mn}$ & -103.4421358 & -104.0762069 & $-104.9731482(1.27)$ & -103.9094048 \\
\hline STUTTGART & & -122.9113522 & -123.6518666 & $-123.8366529(2.49)$ & \\
\hline CRENBL ECP & & -122.5226617 & -122.4547082 & $-123.3195571(3.34)$ & \\
\hline VTZ_ANO & $\mathrm{Fe}$ & -122.7314868 & -123.4474669 & $-124.0565188(1.84)$ & -123.3986474 \\
\hline STUTTGART & & -144.5855681 & -145.0660268 & $-145.694134(3.14)$ & \\
\hline CRENBL ECP & & -143.8138444 & -144.440941 & $-145.1107608(3.60)$ & \\
\hline VTZ_ANO & $\mathrm{Co}$ & -144.5251884 & -145.1652982 & $-145.8621974(2.05)$ & -145.0400758 \\
\hline STUTTGART & & -169.3680007 & -170.0703347 & $-170.7350688(3.83)$ & \\
\hline CRENBL ECP & & -167.8202533 & -168.3037662 & $-169.379119(4.67)$ & \\
\hline VTZ_ANO & $\mathrm{Ni}$ & -168.310308 & -169.3188349 & $-170.5437021(2.81)$ & -169.2595814 \\
\hline
\end{tabular}

Table 2. The total ground state energies computed within Hartree Fock, $E_{\mathrm{HF}}$, variational Monte Carlo, $E_{\mathrm{VMC}}$, and diffusion Monte Carlo, $E_{\mathrm{DMC}}$, for $\mathrm{Sc}$ through Ni cations, the statistical error is in parenthesis. All energies are in Hartree $(\mathrm{H})$.

\begin{tabular}{|c|c|c|c|c|}
\hline Basis Set & Cation & $\mathrm{E}_{\mathrm{HF}}$ & $\mathrm{E}_{\mathrm{VMC}}$ & $\mathrm{E}_{\mathrm{DMC}}(\sigma)$ \\
\hline STUTTGART & & -46.02334453 & -46.34986781 & $-46.38446744(0.95)$ \\
\hline CRENBL ECP & & -45.76732654 & -46.16488456 & $-46.24995164(1.11)$ \\
\hline VTZ_ANO & $\mathrm{Sc}^{+}$ & -45.99434864 & -46.30249107 & $-46.46933261(0.77)$ \\
\hline STUTTGART & & -57.45680287 & -57.85947705 & $-57.91890205(1.25)$ \\
\hline CRENBL ECP & & -57.2889738 & -57.6681311 & $-57.68881859(1.76)$ \\
\hline VTZ_ANO & $\mathrm{Ti}^{+}$ & -57.37217477 & -57.84411887 & $-57.93398356(0.83)$ \\
\hline STUTTGART & & -70.91248923 & -71.20568489 & $-71.26864044(1.41)$ \\
\hline CRENBL ECP & & -70.6473448 & -70.84817829 & $-70.9875304(1.62)$ \\
\hline VTZ_ANO & $\mathrm{V}^{+}$ & -70.8429041 & -71.14789437 & $-71.31218887(1.00)$ \\
\hline STUTTGART & & -86.10780296 & -86.48441035 & $-86.62702495(1.58)$ \\
\hline CRENBL ECP & & -85.49648312 & -86.22461405 & $-86.29249361(1.86)$ \\
\hline VTZ_ANO & $\mathrm{Cr}^{+}$ & -85.89504066 & -86.5588753 & $-86.75599821(1.10)$ \\
\hline STUTTGART & & -103.4810621 & -103.9275429 & $-105.5790461(2.37)$ \\
\hline CRENBL ECP & & -102.8140547 & -103.5044943 & $-104.2741013(2.13)$ \\
\hline VTZ_ANO & $\mathrm{Mn}^{+}$ & -103.3222889 & -103.9555627 & $-104.6990498(1.17)$ \\
\hline STUTTGART & & -122.628094 & -123.246477 & $-123.5537061(2.40)$ \\
\hline CRENBL ECP & & -122.5917666 & -122.8857454 & $-123.0461831(3.12)$ \\
\hline VTZ_ANO & $\mathrm{Fe}^{+}$ & -122.6970404 & -123.4385803 & $-123.7659517(1.32)$ \\
\hline STUTTGART & & -144.9838979 & -145.3994863 & $-145.4024792(3.25)$ \\
\hline CRENBL ECP & & -144.109787 & -144.6034168 & $-144.8391182(3.64)$ \\
\hline VTZ_ANO & $\mathrm{Co}^{+}$ & -143.9132802 & -145.3160708 & $-145.5725396(1.46)$ \\
\hline STUTTGART & & -168.7340016 & -170.0684125 & $-170.4494688(3.36)$ \\
\hline CRENBL ECP & & -167.7769972 & -167.4338226 & $-169.0894278(4.77)$ \\
\hline VTZ_ANO & $\mathrm{Ni}^{+}$ & -168.1397915 & -169.6454674 & $-170.2626752(1.91)$ \\
\hline
\end{tabular}


Table 3. The theoretical and experimental results of the ionization potentials in eVfor incomplete 3d-transition metal neutral atoms for all basis sets.

\begin{tabular}{|c|c|c|c|}
\hline Basis Set & Atom & IP1(Theo.) & IP1(Exp.) \\
\hline STUTTGART & & 6.6806 & \\
\hline CRENBL ECP & & 6.2646 & \\
\hline VTZ_ANO & $\mathrm{Sc}$ & 6.5302 & 6.5615 \\
\hline STUTTGART & & 6.6590 & \\
\hline CRENBL ECP & & 6.7484 & \\
\hline VTZ_ANO & $\mathrm{Ti}$ & 6.87609 & 6.8281 \\
\hline STUTTGART & & 6.7681 & \\
\hline CRENBL ECP & & 6.9601 & \\
\hline VTZ_ANO & V & 6.7602 & 6.7462 \\
\hline STUTTGART & & 6.7434 & \\
\hline CRENBL ECP & & 6.4288 & \\
\hline VTZ_ANO & $\mathrm{Cr}$ & 6.7509 & 6.7665 \\
\hline STUTTGART & & 7.6787 & \\
\hline CRENBL ECP & & 7.4696 & \\
\hline VTZ_ANO & Mn & 7.4582 & 7.4340 \\
\hline STUTTGART & & 7.6990 & \\
\hline CRENBL ECP & & 7.4385 & \\
\hline VTZ_ANO & $\mathrm{Fe}$ & 7.9063 & 7.9024 \\
\hline STUTTGART & & 7.9359 & \\
\hline CRENBL ECP & & 7.3914 & \\
\hline VTZ_ANO & Co & 7.8816 & 7.8810 \\
\hline STUTTGART & & 7.7712 & \\
\hline CRENBL ECP & & 7.8825 & \\
\hline VTZ_ANO & $\mathrm{Ni}$ & 7.6467 & 7.6399 \\
\hline
\end{tabular}

$E_{\mathrm{HF}}, E_{\mathrm{VMC}}$, and $E_{\mathrm{DMC}}$ ground state energies for incomplete 3-d neutral atoms ( $\mathrm{Sc}$ through $\mathrm{Ni}$ ) and their corresponding cations, in parenthesis is the standard deviation (or statistical error), are tabulated in Tabs. (1-2). For the sake of comparison we also give the results of Chiodo et al. [13] who used LANL2DZ $Z_{\text {B3LYP }}$ as basis sets. (Figure 1) Shows that the total ground state energies $E_{\mathrm{DMC}}$ for incomplete 3-d neutral atoms change with the atomic number smoothly.

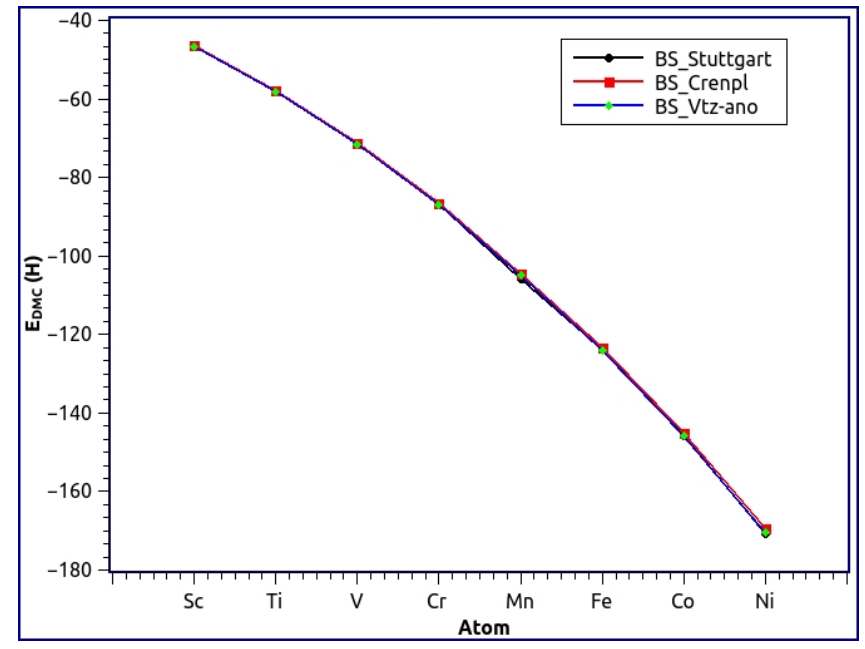

Figure 1. The variation of $E_{\mathrm{DMC}}$ with the atomic number for all basis sets.

As expected, the $E_{\mathrm{VMC}}$ values are a little larger than the $E_{\mathrm{DMC}}$ one; this is arising principally because the accuracy of DMC method is better than VMC one. $E_{\mathrm{HF}}$ is still the worst since $\mathrm{HF}$ approximation neglects the electron-electron correlations [9].

It is clear from (table 1) that our calculations for the neutral atoms of the incomplete 3-d transition elements, for all three basis sets VTZ_ANO [10], STUTTGART and CRENBL ECP [11], are in good agreement with the LANL2DZ ${ }_{\mathrm{B} 3 \mathrm{LYP}}$ results reported by S. Chiodo et al. [13]. Moreover, our calculations is better than those obtained by them, since our results for these atoms is lower than their results $[14,15]$. On the other hand, for the sake of comparison, the results we got with basis set VTZ_ANO are the best in comparison to those with other two basis sets (i.e. STUTTGART and CRENBL ECP). This is because VTZ_ANO basis set is well adapted to quantum Monte Carlo calculations.

\subsection{Ionization Potential}

The ionization potential is a measure of the ionization energy, which is equal to the work expended in emitting the electron from the atom or molecule and characterizes the electron's bond strength in the atom or molecule. The ionization potential is commonly expressed in volts and is numerically equal to the ionization energy in electron volts [16].

In practice, the ionization potential, $\operatorname{IP} 1\left(\equiv I P_{D M C}\right)$, is defined as the difference between the ground state energies of neutral atoms and that of the corresponding cations. We estimated the first ionization potential for Sc through Ni by DMC method using three different Basis Sets, our results al- 
ong with the experimental data from literature [17-19] are tabulated in (table 3) and plotted in Fig. 2.

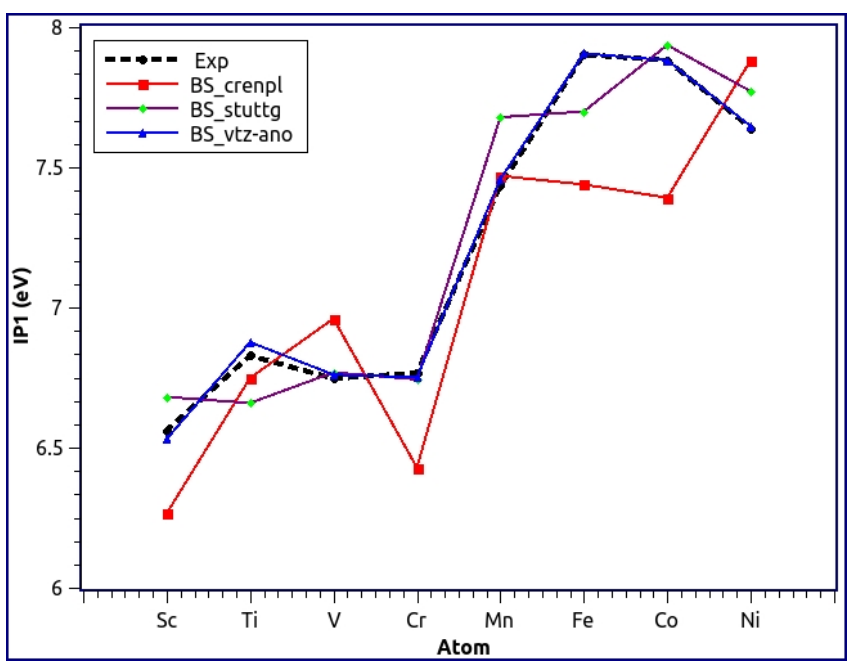

Figure 2. The theoretical and experimental results of the ionization potentials as a function of the atomic number for all basis sets.

The results for IP1 calculated using BS VTZ_ANO are in excellent agreement with the experimental data. This is because BS VTZ_ANO is adapted to quantum Monte Carlo [10], as mentioned before. However, the results of the other two BSs are even in good agreement with the experimental data.

\section{Conclusions}

We studied the incomplete 3d-transition metal elements by means of QMC (strictly speaking DMC) with three basis sets, namely Stuttgart RSC 1997 ECP, CRENBL ECP and VTZ_ANO, and with pseudopotential technique. We found the following:

- The DMC method is adoptable and adaptable, improvable, upgradable and accurate.

- The VTZ_ANO basis set is the most suitable basis set for our destination, then Stuttgart RSC 1997 ECP basis set, at the third place comes the CRENBL ECP basis set.

- The ground state energies and the ionization potential are calculated and compared with theoretical and experimental results (agree very well)

- We got good results for the total ground state energies and ionization potential for all studied neutral incomplete 3d-transition metal atoms and their corresponding cations.

- The software we use, indeed, meets our needs to study some elements by means of Diffusion Monte Carlo method.

\section{REFERENCES}

[1] Mary Bojan, Chapter 22: Transition metals, 2013, online available from http://courses.chem.psu.edu/chem112/Summer/Lecture\%20Notes/12_TransitionMetals_1.pdf

[2] Yinon Bentor, Periodic table: Transition metals, 1996-2012, online available from chemicalelements.com/groups/transition.html

[3] M. W. Schmidt and K. K. Baldridge and J. A. Boatz and S. T. Elbert and M. S. Gordon and J. H. Jensen and S. Koseki and N. Matsunaga and K. A. Nguyen and S. J. Su and T. L. Windus and M. Dupuis and J. A. Montgomery, General Atomic and Molecular Electronic Structure System, J.Comput.Chem., Vol.14, 1347-1363, 1993.

[4] Wagner, Lucas K and Bajdich, Michal and Mitas, Lubos, QWalk: A quantum Monte Carlo program for electronic structure, Elsevier, Journal of Computational Physics, Vol.228, No.9, 3390-3404, 2009.

[5] Chapter 9: Monte Carlo methods, online available from https://www.cs.nyu.edu/courses/fall06/G22.2112001/MonteCarlo.pdf

[6] Matús Dubecký, Quantum Monte Carlo For Noncovalent Interactions: A Tutorial Review, acta physica slovaca, Vol.64, No.5, 501-574, 2014.

[7] Vesa Apaja, Monte Carlo Methods, Johannes Kepler Universit'at, Linz, Austria, 2005, online available from http://users.jyu.fi/ṽeapaja/Monte_Carlo/MC.lecture.-vesa.pdf

[8] Foulkes, W. M. C. and Mitas, L. and Needs, R. J. and Rajagopal, G., Quantum Monte Carlo simulations of solids, American Physical Society, Rev. Mod. Phys., Vol.73, issue 1, 33-83, 2001.

[9] Nagat Mohamed Ibrahim Elkahwagy, Study of Some Quantum Monte Carlo Techniques and its Applications, mathesis, University of Menoufia, Egypt, 2014.

[10] M. Burkatzki and Claudia Filippi and M. Dolg, Energyconsistent small-core pseudopotentials for 3d-transition metals adapted to quantum Monte Carlo calculations, AIP Publishing, The Journal of Chemical Physics, Vol.129, No.16, 164115, 2008.

[11] Schuchardt, Karen L and Didier, Brett $\mathrm{T}$ and Elsethagen, Todd and Sun, Lisong and Gurumoorthi, Vidhya and Chase, Jared and Li, Jun and Windus, Theresa L, Basis set exchange: a community database for computational sciences, ACS Publications, Journal of chemical information and modeling, Vol.47, No.3, 1045-1052, 2007. 
[12] Ceperley, DM, Application of Quantum Monte Carlo to Problems in Electronic Structure, Computer Simulation in Materials Science, Springer, 321-332, 1991.

[13] Chiodo, S and Russo, N and Sicilia, E, LANL2DZ basis sets recontracted in the framework of density functional theory, AIP Publishing, The Journal of chemical physics, Vol.125, No.10, 104107, 2006.

[14] A. Scemama and T. Applencourt and E. Giner and M. Caffarel, Accurate nonrelativistic ground-state energies of $3 \mathrm{~d}$ transition metal atoms, The Journal of Chemical Physics, Vol.141, No.24, 244110, 2014.

[15] R. K. Bhaduri, M. Brack, Atomic ground state energies, Can. J. Phys., Vol.58, 1684, 1980.

[16] M. A. El' iashevich, Ionization Potential, The Great Soviet Encyclopedia, 3rd Edition (1970-1979).
2010 The Gale Group, Inc, online available from http://encyclopedia2.thefreedictionary.com/ionization +potential

[17] Sugar, J. and Corliss, C., Atomic energy levels of the iron-period elements: potassium through nickel, United States, 1985.

[18] E. Buendía and F.J. Gálvez and P. Maldonado and A. Sarsa, Quantum Monte Carlo ionization potential and electron affinity for transition metal atoms, Chemical Physics Letters, Vol.559, 12-17, 2013.

[19] A. Kramida and Yu. Ralchenko and J. Reader and and NIST ASD Team, NIST Atomic Spectra Database (ver. 5.3), National Institute of Standards and Technology, Gaithersburg, MD., online available from http://physics.nist.gov/asd, 2015. 